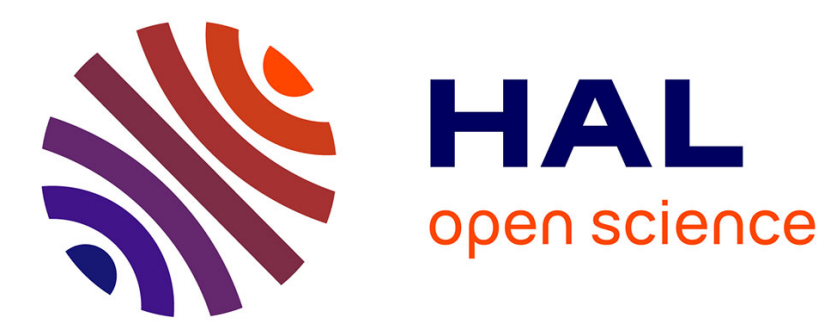

\title{
Participatory Video, Giving Voice and Respect to the Epistemic Sovereignty of Communities in Rural Zimbabwe
}

Peter Makamba, Chido Matewa, Janneke Van Dijk, Gertjan Van Stam, Peter Vhoko

\section{- To cite this version:}

Peter Makamba, Chido Matewa, Janneke Van Dijk, Gertjan Van Stam, Peter Vhoko. Participatory Video, Giving Voice and Respect to the Epistemic Sovereignty of Communities in Rural Zimbabwe. 15th International Conference on Social Implications of Computers in Developing Countries (ICT4D), May 2019, Dar es Salaam, Tanzania. pp.110-121, 10.1007/978-3-030-19115-3_10 . hal-02281320

\section{HAL Id: hal-02281320 \\ https://inria.hal.science/hal-02281320}

Submitted on 9 Sep 2019

HAL is a multi-disciplinary open access archive for the deposit and dissemination of scientific research documents, whether they are published or not. The documents may come from teaching and research institutions in France or abroad, or from public or private research centers.
L'archive ouverte pluridisciplinaire HAL, est destinée au dépôt et à la diffusion de documents scientifiques de niveau recherche, publiés ou non, émanant des établissements d'enseignement et de recherche français ou étrangers, des laboratoires publics ou privés.

\section{(c)(1)}

Distributed under a Creative Commons Attribution| 4.0 International License 


\title{
Participatory Video, giving Voice and Respect to the Epistemic Sovereignty of communities in rural Zimbabwe
}

\author{
Peter Makamba ${ }^{1}$, Chido Matewa², Janneke van Dijk ${ }^{3}$, \\ Gertjan van Stam $^{3}$, Peter Vhoko ${ }^{4}$ \\ ${ }^{1}$ Apostolic Church, Bikita, Zimbabwe \\ ${ }^{2}$ Africa Women Filmmakers Trust, Harare, Zimbabwe \\ ${ }^{3}$ SolidarMed, Masvingo, Zimbabwe \\ ${ }^{4}$ Zimbabwe National Family Planning Council, Masvingo, Zimbabwe \\ authors in alphabetical order, corresponding author: gertjan@vanstam
}

\begin{abstract}
To inform program development in health in the rural areas of Masvingo Province in Zimbabwe, Ministry of Health and Child Care authorised cooperating partners to execute participatory video projects in rural districts in Masvingo Province. The Participatory Video process was a result of the desire to go beyond prescribed, non-participative or cultural insensitive data gathering methods. Two videos were produced that significantly shaped thinking of stakeholders being informed on health-seeking behaviours and utilisation of services. This use of Information and Communication Technologies proved a fruitful way to engage, interact, and develop public conversations, giving a voice to formerly disenfranchised groups (e.g. ostracised religious groups or 'closed' communities'). Participatory Video is recognised as a culturally aligned method appropriate for a setting other than the West.
\end{abstract}

Keywords: Participatory video, health, Zimbabwe

\section{Introduction}

Health research in the context of African rural communities in sub-Saharan Africa often uses extraneous approaches that leave little space for indigenous, rural perspectives $[1,2]$. Long established and prescriptive methods tend to pre-organise and regurgitate topics and practices. Most data gathering methods used are not participatory, with limited community involvement. Further, data collecting procedures observe a distance between the researcher and the researched. In practice, such approaches can be perceived as alienating, as the methods rely upon normative epistemologies that obscure local, dynamic and integral ways of knowing [3]. The authors engaged in an effort to push the boundary of the established ways of information gathering for health, utilising Information and Communication Technologies in the form of media. The engagement aimed to unlock local knowledge and indigenous perspectives to provide evidence on possible contentious subjects to inform health planning. In this paper, authors represent groups of community members and present the background, the context, and the cases and lessons learned from utilising participatory video in a health context. 
Although Participatory Video (PV) already emerged in the 1980s, its existence and employ were unknown to the communities and cooperating partners in health care in Masvingo Province. PV utilises ICTs to facilitate community members to express themselves in ways they are used to: in orality. Some of the authors were sensitised to the existence of PV, having been involved in the method in Macha, Zambia, years ago. Activating the method in health became possible after hearing about activities in PV by a group of women in Zimbabwe. Cooperating partners agreed to trial the technique for matters related to maternal and sexual reproductive health care; to gather evidence on local perceptions on family planning and care surrounding deliveries, and for exploring the practical experiences and barriers to related health care in their geographical areas.

This paper uses the extended case method to study the PV cases presented here, by valorising the intervention, process, structuration, and theory as they live in their extra-local and historical context [4]. We explain how PV was applied ethically and fruitfully to create a community-led information base as a repository for change in the quality of care by presenting two consecutive cases of the use of PV in 2016 and 2017.

\section{Participatory Video, a theoretical-practical overview}

Participative Video was proposed as a tool that respects self-determination across connected identities and dimensions within community life. It represents a method that allows disenfranchised people to be the authors, architects and arbiters of their development [5]. The method provides means to access narratives and content that communities ascribe to their situation. Shaw and Robertson [6] situate participatory video "as a social and community-based tool for individual and group development [...] to develop their confidence and self-esteem, to encourage creative selfexpression, and to develop a critical awareness". The first documented participator film process, at Fogo Island, Newfoundland, in 1967, used an iterative process of production and multiple screenings to an aggregated audience of 3,000 persons in isolated communities [7].

Participatory processes provide means for communities to communicate among themselves and with other communities of practice, in this case in health care. It allows community members to be collective prosumers - being both producers and consumers - in the production and incorporation of data, analysis, and knowledge. PV was considered a method appropriate for settings other than the West [2]. PV allows for community engagement [8], the first step in a three-step strategy for access to services, with the others being workforce development and thought leadership [9]. The developments emerging from community engagement are distinctly different from most empowerment approaches that target the economic situation of individuals using externally, non-African developed schemas [10]. With outcomes in the form of recorded narratives, PV unearths collective knowledge - that what is integrally known in a community in embodied formats [3, 11, 12]. 
The participatory video method aligns with Freire's [13] arguments that disadvantaged people groups must be the principal protagonists to engender transformative change. Participative Video is participatory in that those involved are actively engaged participants and actors rather than 'objects of observation' and 'sources of data' [14]. It avoids extractive research, where the primary target is the increase in knowledge in an external researcher [15]. Participative Video represents a form of transdisciplinary, multilevel action research that is respectful of ecological and psycho-political aspects [16]. It seeks out human-centred conceptualisation of development in line with the position of Tanzanian President Julius Nyerere [17] who argued that "people can't be developed, they can only develop themselves."

As a technique, Participatory Video was approached as a process and not an event. The lengthy process empowers communities, facilitating ownership of the activities and the outcomes. Contemporary reports of PV practices mainly discuss cases where cameras are being provided or used by people of the community. This situation allows community members to produce works from local agency 'reading reality through the camera'. For instance, Tony Roberts [7] describes such a PV process applied by Asikana Network in Zambia. In the cases presented in this paper shows that PV is not necessarily a technical process. It is a social one, where the complex role-plays with stakeholders, communities, and national authorities interface with the use of technologies.

\section{Two Cases of PV in Masvingo Province}

The first case in this paper is "Khuluma", a documentary film that highlights the causes of unmet need for family planning in Bikita District, Masvingo Province, Zimbabwe in 2016. A second film was produced to determine the reasons why some women deliver at home. This PV took place in the neighbouring Zaka District in 2017.

The facilitation and resourcing of the PV process were facilitated through a Public Private Community Partnership. The partners are a national organisation with PV facilitators and experts (Africa Women Filmmakers Trust, AWFT), the Ministry of Health and Child Care, and a Private Voluntary Organisation (SolidarMed). AWFT is a Trust and Arts Organization registered with the National Arts Council of Zimbabwe. The collective of women pioneered the use of participatory video in Zimbabwe during the 1990s [5]. SolidarMed a Private Voluntary Organisation (PVO) in Zimbabwe with a supporter base in Switzerland and Liechtenstein. The organisation cooperates with the Government of Zimbabwe for over 50 years. In daily practice, the PVO focuses on the support and execution of Zimbabwe's National Health Strategy through activities in HIV and TB care, Maternal and Newborn Health, Non-Communicable Diseases, and eHealth. The Ministry of Health and Child Care is the government ministry responsible for health in Zimbabwe.

In an effort to gather fresh evidence beyond enshrined methods of informing health care planning, Participatory Video was selected because of its engagement in community dialogues and community conversations, right where community 
members live. PV captures conversations on health that can include cultural, social, economic, religious and service provider aspects which are difficult to obtain through other means and cannot be found in spreadsheets. By amplifying the 'local voice', the partners hypothesised that PV allowed for epistemic sovereignty to all stakeholders in the cascade of health care provisioning and access to the vocalised communal and embodied knowing [11].

After several months of preparation and stakeholder engagement, the community interactions for the first case using PV took place during September and October 2016. The women of AWFT facilitated both process and content when they recorded focus group discussions, non-structured interviews with community members in Bikita district, and semi-structured interviews with health care professionals at the local, provincial and national levels. The second PV process took place the following year, with recordings being done during September and October 2017. This PV applied the lessons learned during the first PV process. Instead of a focus in one area, contributions were gathered from a wider area, at selected sites scattered over Zaka district and, consecutively from academia in Zimbabwe providing a precise framing. Consent was sought for the videos to be used as a pinpointed facility to inform communities, stakeholders and policymakers, respecting the sequencing and hierarchy of stakeholders [18]. Therefore, the results are not made generally available or put on the internet.

\subsection{Process}

Although a Participative Video process is theorised as 'free-flowing', the complex networking of stakeholders and partners and cultural codings necessitates a purposeful embedding of activities. To ensure 'all aboard', both in the health chain (e.g. government agencies at the national, provincial and local level) and the communities (e.g. chiefs, headmen), the process focused on the incorporation of all guidance and the securing of all appropriate formal and informal permissions. This lengthy and involving process focused on:

- involving all service providers and policymakers to include their considerations for the PV process, by seeking continuous guidance through visits and updates

- the extensive use of embodied knowledge in local leaders - e.g. traditional and religious leaders - to structure access, location, and invitations for involvement

- empowerment of communities and individuals to identify and challenge cultural, religious and societal norms, using PV's iterative processes

- the creation of a collective of stakeholders and communities who act together along the way, for instance, through an all-inclusive and public launch of the video.

Africa Women Filmmakers Trust is, to our knowledge, the only organisation in Zimbabwe facilitating Participatory Video processes. They use PV as a development and intervention tool, facilitating the PV process and the production of the final video. AWFT facilitates community gatherings and the operating of the media equipment. Continuous attention is given to content decisions to be taken by the community and ensuring active community gatherings by monitoring of group dynamics in manners 
respectful to local culture and contextual governing structures. The results provide different evidence on how the community understands their domestic situation, often better than outsiders [5]. Hence, through PV, the community contributes their collective knowing and their view on problems and challenges, therewith engaging in the health planning processes.

The PV process, in the two cases, presented, built upon three main pillars:

1) expository videos and written report on process and content, systematically drawing attention to the nature and extent of the subject matter

2) community participation and sensitisation, enabling other communities to use the videos to hold a mirror to themselves, and

3) skill and experience in operating both at the grassroots level and policy advocacy level, ushering the possibility of justice and change.

Receiving access to communities in rural Zimbabwe is laborious. Custom requires adherence to complex and reiterative protocols of respect and reciprocity. As mentioned, at the start of the PV projects, the use and the content of the method and its practices were unknown in the communities and stakeholders. Therefore, the projects took long periods of introduction and sensitisation on the method and process, involving many meetings with governing authorities and the communities involved. During these periods, the focus was gaining and establishing trust. At provincial and district levels, permissions were sought from government authorities as well so-called traditional leaders in line with their authority in their respective areas. In rural communities, informed consent does not necessarily relate to a project or a specific set of questions but is given for the people involved as a validation of their credibility [19]. In the process, the AWTF facilitators were required to obtain press cards.

\subsection{Content}

In the preparations leading up to community encounters, a basis of understanding about the PV method was established in all stakeholders and collaborators. Developments included extensive reading and preliminary research on the literature on the subjects' matter and learning extensively about the contexts. Semi-structured interviews were videoed with academic stakeholders at the national level. These interactive and elaborate preparation processes ensured a framing for the result. Broad cooperation and interaction proved crucial for alignment during communications with stakeholders and authorities [cf, 18].

Gender and age are critical factors that impact access to people groups and leaders, especially in rural areas in Zimbabwe. Therefore, process and execution were managed by Zimbabwean women. Female leadership is conducive for both female and male voices to be able to express their views, while the opposite might not be the case. The inclusive socialities of the rural Zimbabwean communities are embedded in Ubuntu/Unhu, the epistemology emerging from an African paradigm [20, 21]. The inclusive and convivial values of the communities led the PV discussions to gravitate to the subject matter studied by the cooperating partners involved. 
After been introduced and getting to know people, local ways of knowing are revealed to those involved in and co-habituate in the community, over time and through the stories shared by others in the vicinity [11]. For instance, in one of the cases, during familiarisation in the communities, some women belonging to African churches brought up how some religious groups seem to discourage 'modern' (Western-constructed) health care seeking. As a result of this sensitisation, the AWFT strove to include members and leaders from religious groups in the PV process. The targeted inclusion of apostolic religious groupings ensued previously unrevealed evidence of neglected perspectives. For instance, in the case of the PV on family planning, apostolic women explained how they were accessing family planning services creatively. From the same group, in the case of PV on why women deliver at home, women told how they would mediate their health care by periods of geographic separations, especially near the period of delivery. The process and inclusion provided unique insights into how community members manage their multiple identities, for instance, being community members, members of religious groupings, and being travellers. They narrated about switching paradigms, aligning with local realities in, for example, religion or secular concepts of health care in the demanding context of rural community members in Zimbabwe.

During the process, Participative Videos were produced involving discussions during both large community gatherings and in smaller group settings. The setting up and execution of such meetings were done following the local, cultural practices and protocol. In the two cases, the PV method was applied during church meetings, gatherings of women at sheltered waiting areas, at men discussion meetings, or during meetings in the community or with spiritual leaders. Also, the PV process involved the governmental health care providers, officials, and academic stakeholders wherever they were positioned, at a local, district, provincial or national level.

No representatives of any of the cooperating partners were present during introductions and PV recording processes in the rural communities. The AWTF facilitators positioned themselves as Zimbabwean compatriots and as experts in the operations of media equipment like the video camera or light sources. The absence of outsiders was intentional to minimise sources of influence to the community deliberations. Assimilation of PV in the community was to alleviate, as much as possible, external control and cultural biases affecting the language, content and form of deliberations [cf, 12]. All conversations were made in the first language in the community: Shona. The expert team stayed in the community and was included in the daily routines and rhythms of community life.

Participatory Video captures both the content and the interactions in manners set by the community. The subjects that are discussed are the subjects that the community has reason to value. PV allows for multiple approaches, ranging from communities determining both the themes and questions to pre-set themes or questions. In the two cases described here, the context was proposed by a coopering partner to align with its planning for work in support of the Ministry of Health and Child Card in Maternal Neonatal Health support. However, beyond this meta-frame, the AWTF experts and the communities were given no guidance as to what or how to discuss. Therefore, the PVs were developed within the communities according to conventional, local 
practices. Subsequently, the content addressed issues of shared concern. In the second $\mathrm{PV}$ on home deliveries, the framing of the final video was synced with inputs from academics in the national university, aligning the content with academic models gleaned from national research by African researchers. The final product, therefore, provided scientific veracity that bolstered acceptance at the stakeholder level.

The videos triggered lively dialogues during the numerous screenings of the resulting video at community meetings, just like was reported in Fogo. These public conversations were lively due to a recognition of the views expressed in the videos. During sensitisation meetings, communities build upon the work by adding specific views, insights and expand on the issues triggered upon by the videos. PV thus accelerates the processes of learning by providing evidence at different levels of consciousness of the various stakeholders. Such differentiation of views came out emphatically during the official launch of one of the videos. This video provided evidence that few health service providers were trained in long-acting reversible contraceptives. The necessity of such training was taken up during the launch, and included in future health program planning, indicating how 'community knowledge' became 'stakeholder knowledge’.

\section{Discussion}

The Participative Video method aligns with observations from communications professor Francis Nyamnjoh. In his assessment of Journalism in Africa, Africa in Journalism, Nyamnjoh [22] cites Servaes and Arnst who argue that it is about time the poor and the illiterate became actively involved. They "have always been researched, described and interpreted by the rich and educated". Servaes and Arnst argue the poor and the illiterate should take over the research on their predicaments, especially, as often, "they best know their situation and have a perspective on problems and needs that no outsider can fully share”. PV can be instrumental to such epistemic justice, where it relies upon local community dialogues and community conversations that are effusive, addressing broad subjects expansively. In the two cases, the inclusive process substantiated the local understanding of a variety of cultural, social, economic and religious causes of unmet family planning needs and home deliveries. The videos exposed how, according to the community, related issues interplay at a community level. Uniquely, it made the voice heard of (religious) groups in rural communities that are commonly not included in community dialogues.

The PV approach encouraged participants to share experiences, provide observations and relate narratives with insights on content and future scenarios. These insights could well remain undisclosed using other methods, and, thus, be overlooking in the planning of health care interventions. The public ways of information sharing that the PV method calls for are crucial to sustaining an embedded, unpressured way of evidence gathering. The process allows for involvement and scrutiny by all. The process, therefore, allowed for rural communities to discuss contentious and potentially sensitive health issues. This empowered communities to contribute to the 
professional discourse and pinpoint previously underrated issues that affect familyplanning in Bikita district and why women deliver at home in Zaka district.

In the rural setting in Zimbabwe, we found that community members value a quality-of-the-interaction. Adherence to cultural scripts was essential. The process, therefore, supersedes addressing of content. Adhering to the convivial principles of appreciative inquiry - being asked 'nicely'- enabled communities to disclose ways and content of their knowing. Community members were remarkably free, in-depth and open about their perceptions of social, cultural, religious influences and guidance. Also, they were open about their perceptions of behaviour and attitude of health service providers. In the process, poignant aspects and significant influences concerning the subjects' matter were put in the limelight. The PV process provided tangible and stark evidence on the content of beliefs, material realities and socialisations in rural communities. The videos sparked lively debate on the content of the videos both in the institutionalised health chain as in the communities. The videos proved to be a sensitisation tool on the subject matter in consecutive community screenings. The PV method, therefore, showed value in providing visible and readily accessible input for change processes. The tangible evidence offers a base for informed policy and subsequent potential change in programmatic areas.

As PV involves the rendering of a vast repository of video material into a relatively short video, its tangible outcomes provide both lean and thick descriptive evidence. Both forms of evidence proved substantial for ongoing change processes to improve local health care provisioning and systems continuously. The videos contain evidence from communities affirming their views on subject matters within the existing cultural realities. The method unsubalternising the local view, showing individual community members display an appreciation of contemporary realities and indicate the perceived benefits of specific measures of intervention. At the same time, there is a useable source showing a local narrative and comprehension of particular interventions, indicating the local understanding of realities, as well as the local, cultural processes involved with health care decision making. For instance, the videos show bounded ambivalence about the reasoning and intended outcomes of specific interventions. The ongoing showing of the videos in communities in Masvingo province sparked inclusive debates along a continuum from 'closed' via 'arrested' to 'open' to conversion.

During the described PV cases, processing of the orally stored information within the community was done in a verbal equivalent of 'social networks' [cf., 12]. In the process of producing video compilations, community members and stakeholders interacted in the framing of the data. Outputs, therefore, were more helpful in communicating to both the local and the broader contexts of health care in (rural) Zimbabwe. The result provided input for subsequent community meetings in other communities, evoking a response to the subjects' matter. These screenings ensured continuous production of embodied knowledge and unearthing of a public knowing.

Participative Video bridges the insider/outsider segregation. Research methods, for instance, using surveys or the handling of aggregated data, position the researchers as outsiders. Such a position is, often, assumed necessary for objectivity and neutrality. However, in rural areas of Zimbabwe, communities engage in continuous, inclusive 
conversations, from which regularly tested conclusions are drawn [12]. Communities commented positively on researchers participation in community life and talks.

The tangible expression of the result of the PV processes is a product set in an oral format, albeit digitalised. The oral form aligns with how information is starting to permeate within a rural community. Oral culture relies upon continuous conversation, often in scripted manners. The conversation is the knowing [11] which structures restrict experimentations or adding new information without communities' involvement. The process of 'orally' processing new information' by itself is a tool for community engagement and acceptance [12, 23]. The ways of information and knowledge conceptualisation align with the ways that Participatory Video processes deal with information. These emerge naturally through the process of verbalisation while, often, incorporating aspects of the immediate, familiar environment. As such, a 'new world' becomes assimilated into the 'old world'. For instance, high-technology interventions, like the introduction of ICT or an aeroplane, are described in a context of events involving people [24]. This assimilation correlates with the fact that oral culture, in general, does not include counts, statistics or facts, but, instead, keeps track of activity or activities in which humans are involved [25]. The process of PV, recording the verbalisation of community perspectives, let to an opportunity to analyse and discuss the information with the community itself.

The resulting videos were intended to align partners and communities in their focus of health interventions and to promote advanced quality of care in the subject areas. The PV process provided affordances (allows and enables) for expressions in the local language, local ways of doing, without intruding external parties. Taking the six affordances of Tony Roberts [7], the movies allowed for:

- Reflection. Respectful to the primacy of the local community, PV created the space for local reflections on the subject matter and the valuing of local perspectives. Secondly, through the execution of 'official launches' of the videos, stakeholders and those involved in the video reflected on the content, openly. Thirdly, the broader health community in Zimbabwe use the videos to reflect and assess the potential to improve quality of care. The PV process in itself, brought conscientisation for all involved as this was unusual - though culturally aligned manner of being confronted with information and bringing knowledge into a broader realm.

- Engagement. In one of the video activities, the PV process, unexpectedly, brought the views of a religious grouping to the fore. This development was appreciated as opening contemporary facilities to enhance communications. Previously, interactions with, especially, so-called indigenous African churches were strained in the context of 'modern' health care. Through the PV process, the engagement of all involved was appreciated, including members and leaders of African churches. Through these engagements, various views became accessible and became seeds to surmount barriers of institutional ignorance or incomprehension.

- Dialogue. Respectful to the primacy of the local language, the PV method allows for interactions in the vernacular language, Shona in these cases. Allowing the process to be in the local language from start to finish strengthened 
communications in a situation where 'normally' conversation take place 'in translation' [26]. Stories and words used in the videos allowed for proper depth and cultural consciousness that sustains an impact beyond the normal 'text focused' social-political-culture structures in healthcare research and dissemination.

- Intent. The PV method builds upon a shared intention to allow a collective voice to be expressed. Structuring processes in tune with local cultures brought to light local ways of dealing with the subjects at hand. Such input validated the complex and in-depth local knowledge and perceptions, its systems of knowing, and sets the scenes for change.

- Voice. PV alleviates the conundrum of how to accord 'voice to the rendered voiceless' [27], In the process, due diligence is given to issues of language, the identification and categorisation of local problems and their articulation in context. Further, the videos provide insights into the contemporary and shifting local priorities, the critical, local questioning of common knowledge and the addressing of taboos.

- Action. The video output of the PV process travels quickly, allowing for its agency to be felt widely. Although controlled by stakeholders, the video 'artefact', can move on USB sticks, exerting influence in a fluid and helpful way.

The Participatory Video process was particularly compelling to engage the community. Engagement, of course, took much time and effort. The whole process took about half a year each. In that manner, it proved difficult to reconcile this fluid and qualitative method in the quantitative focused health care planning processes. However, the commitment to engage allowed inclusion and active involvement of what would be considered 'closed communities', where thinking is considered to be set in strict value systems without much agency for change [23]. Access to various religious groups is deemed very difficult as they are depicted as 'closed'. Subsequently, their 'underutilisation' of 'modern health care' has been criticised [28]. However, the leadership of a large religious grouping in Bikita embraced the participatory video process, and, subsequently, contributed significantly. Through the inclusiveness and openness of PV, members of all groups in the communities could participate and engage in discussions.

\section{Conclusions}

This extended case study of the use of Participative Video shows its feasibility in rural Zimbabwe. In an environment where the method was unknown, it succeeded to provide input into health planning processes through a representation of the local voice and showing respect to the epistemic sovereignty of communities in rural Zimbabwe. The method unearthed collective knowledge and validated Zimbabwean academic models contributing material information in a new format for a health care development environment 'set in its ways'. The method succeeded to carry input from a context in culturally appropriate ways to the stakeholders involved in (provincial) health planning in Zimbabwe. Community views provided inputs that were previously 
subalternised and, therefore, relatively unknown to those facilitating public health interventions and health care. From the two case studies presented, we conclude that Participative Video is a method that facilitates information gathering that increases social and political engagement of groups in rural Zimbabwe through a means of community engagement. The videos continue to raise awareness about the local views on the matter being discussed. They provide entry points in conversation through subsequent showing in other communities. By allowing the voice to marginalised communities, PV allows for social-emotional learning that expands beyond the ideas presented in the videos. Also, the videos have a transformational agency as they frame the follow-ups in the designs of the related health intervention program in rural communities.

The PV process facilitation was done by Zimbabwean women, allowing for the participation of all genders while embedding access control to tools and framing within the context of the areas involved. The cases show how Participatory Video is instrumental in improving and deepening healthcare practices in alignment with 'local ways of knowing' and the cultural and political realities of the Zimbabwean, rural context. The PV process, though, is extensive and caters for involvement instead of 'token participation'. PV exposes local perspectives on complex social issues and reveals subjective, emotional and dynamic factors in rural communities, also on sensitive topics. It is a method to bring community voices to the fore, not to solve health issues there and then, and therefore provides for qualitative inputs. The process can engage local, rural communities, health care providers and decision makers at district, provincial and national levels in Zimbabwe to engage subsequent communities and inform policy and program design aligned with the realities in context.

\section{References}

1. Mawere, M., van Stam, G.: Research in Africa for Africa? Probing the Effect and Credibility of Research Done by Foreigners for Africa. In: IFIP WG 9.4 Conference, 1-3 May 2019, Dar es Salaam, Tanzania (2019).

2. van Stam, G.: Method of Research in a We-Paradigm, lessons on Living Research in Africa. In: IFIP WG 9.4 Conference, 1-3 May 2019, Dar es Salaam, Tanzania (2019).

3. Bigirimana, S.: Beyond the thinking and doing dichotomy: integrating individual and institutional rationality. Kybernetes 46, 1597-1610 (2017).

4. Burawoy, M.: The Extended Case Method. Sociological Theory 16, 4-33 (1998).

5. Matewa, C.: Media and the empowerment of communities for social change. University of Manchester, Manchester (2003).

6. Shaw, J., Robertson, C.: Participatory video: A practical guide to using video creatively in group development work. Routledge, London (1997).

7. Roberts, T.: Critical-Agency in ICT4D: a case study of Zambian women's use of participatory video technology to challenge gender inequality. Royal Holloway, London (2015).

8. Bishi, J., Shamu, A., van Dijk, J., van Stam, G.: Community Engagement for 
eHealth in Masvingo, Zimbabwe. In: Proceedings of $1^{\text {st }}$ International Multi Disciplinary Conference, 23-25 August 2017, Lusaka, Zambia (2017).

9. van Stam, G.: A Strategy to make ICT accessible in rural Zambia: A case study of Macha. Nelson Mandela Metropolitan University, Port Elizabeth (2013).

10. Tandon, R.: Participation, citizenship and democracy: reflections on 25 years of PRIA. Community Development Journal 43, 284-296 (2008).

11. Mawere, M., van Stam, G.: Oratio: A Framing of Knowledge in the Context of Technology and Academia. In: Mawere, M. Mubaya, T. (eds.) African Studies in the Academy. The Cornucopia of Theory, Praxis and Transformation in Africa?, pp. 251-264. Langaa RPCIG, Bamenda (2017).

12. van Stam, G.: Information and Knowledge Transfer in the rural community of Macha, Zambia. The Journal of Community Informatics 9 (2013).

13. Freire, P.: Pedagogy of the Oppressed. Continuum International Publishing Group, New York (2000).

14. Chambers, R.: Rural development: Putting the Last First. Longman, Essex (1983).

15. Fals-Borda, O., Rahman, M.: Action and Knowledge: Breaking the Monopoly With Participatory Research. Apex Press, New York (1991).

16. Christens, B., Perkins, D.: Transdisciplinary, multilevel action research to enhance ecological and psychopolitical validity. Journal of Community Psychology 36, 214-231 (2008).

17. Nyerere, J.: Freedom and development: Uhuru na maendeleo. A selection from writings and speeches 1968-1973. Oxford University Press, Oxford (1973).

18. Kroczek, A., Mweetwa, F., van Stam, G.: Stakeholder Theory and ICT in rural Macha, Zambia. In: Proceedings of $5^{\text {th }}$ Annual International Conference on ICT for Africa (ICT4Africa), 20-23 Feb 2013, Harare, Zimbabwe (2013).

19. Smith, L.: Decolonizing Methodologies. Research and Indigenous Peoples. Zed Books, London, New York (1999).

20. Metz, T.: A Life of Struggle as Ubuntu. In: Ndlovu-Gatsheni. S., Ngcaweni, B. (eds.) Nelson Ndlovu-Gatsheni: Decolonial Ethics of Liberation and Servant Leadership, Africa World Press, Trenton (2015).

21. Mawere, M., van Stam, G.: Ubuntu/Unhu as Communal Love: Critical Reflections on the Sociology of Ubuntu and Communal Life in sub-Saharan Africa. In: Mawere, M., Marongwe, N. (eds.) Violence, Politics and Conflict Management in Africa: Envisioning Transformation, Peace and Unity in the Twenty-First Century, pp. 287-304. Langaa RPCIG, Bamenda (2016).

22. Nyamnjoh, F.: Journalism in Africa, Africa in Journalism. Rhodes Journalism Review, 3-6 (2005).

23. van Stam, G.: Inclusive Community Engagement in Social Innovation, case Africa. In: Fifth International IEEE EAI Conference on e Infrastructure and e Services for Developing Countries (Africomm 2013), 25-27 November 2013, Blantyre, Malawi (2013).

24. van Stam, G.: Reflections: a narrative on displacement of technology and meaning in an African place. Gertjan van Stam, Harare, Masvingo, Macha, Tilburg (2017). 
25. Ong, W.: Orality and Literacy. The Technologizing of the Word. Routledge, London, New York (1982).

26. Ezema, P.: Semiotic translation and the expression of African thoughts and cultural values in English. Journal of African Studies and Development 5, 218-223 (2013).

27. Spivak, G.: Can the Subaltern Speak? In: Nelson, C., Grossberg, L. (eds) Marxism and the Interpretation of Culture, pp. 66-111. University of Illinois Press, Champaign (1988).

28. Abdul-Karim, C.: "There is no development here": Social Power and the Shaping of a Southern Zambian Community. Washington State University, Seattle (2012). 